\title{
Perfil Epidemiológico dos Casos de Violência Infantil em Escolas Municipais de Ensino Básico
}

Epidemiological Profile of Child Violence Cases in Municipal Schools of Basic

Perfil Epidemiológico de los Casos de Violencia Infantil en las Escuelas Primarias Municipales

Cléa Adas Saliba GARBIN

Programa de Pós-graduação em Odontologia Preventiva e Social, UNESP - Universidade Estadual Paulista, 16015-050 Araçatuba - SP, Brasil https://orcid.org/0000-0001-5069-8812

Tânia Adas SALIBA

Programa de Pós-graduação em Odontologia Preventiva e Social, UNESP - Universidade Estadual Paulista, 16015-050 Araçatuba - SP, Brasil https://orcid.org/0000-0003-1327-2913

Naiana de Melo BELILA

Programa de Pós-graduação em Odontologia Preventiva e Social, UNESP - Universidade Estadual Paulista, 16015-050 Araçatuba - SP, Brasil https://orcid.org/0000-0002-1099-9116

Ana Victória BUTARELO

Programa de Pós-graduação em Odontologia Preventiva e Social, UNESP - Universidade Estadual Paulista, 16015-050 Araçatuba - SP, Brasil https://orcid.org/0000-0003-1145-7607

Maria Clara Faria POLI

Programa de Pós-graduação em Odontologia Preventiva e Social, UNESP - Universidade Estadual Paulista, 16015-050 Araçatuba - SP, Brasil https://orcid.org/0000-0003-4601-4160

Artênio José Ísper GARBIN

Programa de Pós-graduação em Odontologia Preventiva e Social, UNESP - Universidade Estadual Paulista, 16015-050 Araçatuba - SP, Brasil https://orcid.org/0000-0002-7017-8942

\section{Resumo}

Introdução: A violência infantil é praticada das mais diversas formas, entre elas a física, a psicológica, a sexual e as situações de negligência. Os sinais e sintomas das vítimas podem ser vistos também em ambientes extrafamiliares, como por exemplo, na escola, uma vez que o contato com criança é maior do que em qualquer outro serviço. Objetivo: Identificar casos suspeitos e/ou confirmados de violência contra crianças em escolas municipais de Ensino Básico em um município de médio porte do Estado de São Paulo. Metodologia: Trata-se de um estudo descritivo, de caráter transversal e abordagem quantitativa realizado em 5 escolas no período de agosto de 2018 a abril de 2019. A identificação dos casos suspeitos e/ou confirmados de violência contra crianças foi efetuada por meio de um inquérito, respondido pelos professores das instituições. Resultados: Identificaramse 90 casos de agressão, onde $57,8 \%$ ocorrem em crianças do sexo masculino, com idade de 5 anos (27,8\%). A maioria dos agressores foram os pais (72\%), onde $74 \%$ foram casos de negligência, sendo o piolho (42,2\%) e a cárie dentária (34,5\%) como as principais causas. Do total de casos, $46,7 \%$ foram reincidentes. Conclusão: Conclui-se que muitas crianças vêm sofrendo com algum tipo de agressão doméstica e que os sinais e sintomas das vítimas podem ser observados também em ambientes extrafamiliares, como por exemplo, a escola.

Descritores: Maus-Tratos Infantis; Violência; Educação Infantil.

\section{Abstract}

Introduction: Child violence is practiced in the most diverse ways, including physical, psychological, sexual and negligent situations. The signs and symptoms of the victims can also be seen in extra-family environments, such as at school, since contact with children is greater than in any other service. Objective: To identify suspected and / or confirmed cases of violence against children in municipal elementary schools in a medium-sized municipality in the state of São Paulo. Methodology: This is a descriptive, cross-sectional study with a quantitative approach carried out in 5 schools from August 2018 to April 2019 . The identification of suspected and / or confirmed cases of violence against children was carried out through a survey, answered by the teachers of the institutions. Results: 90 cases of aggression were identified, of which $57.8 \%$ occur in male children, aged 5 years $(27.8 \%)$. Most of the aggressors were the parents $(72 \%)$, where $74 \%$ were cases of negligence, with the louse $(42.2 \%)$ and tooth decay (34.5\%) as the main causes. Of the total cases, $46.7 \%$ were repeat offenders. Conclusion: It is concluded that many children have been suffering from some type of domestic aggression and that the signs and symptoms of the victims can also be observed in extra-family environments, such as at school.

Descriptors: Child Abuse; Violence; Child Rearing.

\section{Resumen}

Introducción: La violencia infantil se practica de las maneras más diversas, incluidas las situaciones físicas, psicológicas, sexuales y negligentes. Los signos y síntomas de las víctimas también se pueden ver en entornos extrafamiliares, como en la escuela, ya que el contacto con los niños es mayor que en cualquier otro servicio. Objetivo: Identificar casos sospechosos y / o confirmados de violencia contra niños en escuelas primarias municipales en un municipio de tamaño mediano en el estado de São Paulo. Metodología: Este es un estudio descriptivo, transversal con un enfoque cuantitativo llevado a cabo en 5 escuelas desde agosto de 2018 hasta abril de 2019. La identificación de casos sospechosos y / o confirmados de violencia contra los niños se realizó a través de un encuesta, respondida por los docentes de las instituciones. Resultados: Se identificaron 90 casos de agresión, de los cuales $57.8 \%$ ocurrieron en niños varones de 5 años (27.8\%). La mayoría de los agresores fueron los padres $(72 \%)$, donde el $74 \%$ fueron casos de negligencia, con el piojo $(42,2 \%)$ y la caries dental $(34,5 \%)$ como las principales causas. Del total de casos, el $46.7 \%$ fueron reincidentes. Conclusión: Se concluye que muchos niños han sufrido algún tipo de agresión doméstica y que los signos y síntomas de las víctimas también se pueden observar en entornos extrafamiliares, como en la escuela.

Descriptores: Maltrato a los Niños; Violencia; Crianza del Niño.

\section{INTRODUÇÃOO}

A violência é colocada como um fenômeno multideterminado e multifatorial que há anos está presente na sociedade ${ }^{1}$.É apontado como um problema social e de saúde pública, pois tem influência direta na qualidade de vida das vítimas podendo ocasionar lesões psíquicas, físicas e morais ${ }^{2}$.
Em determinadas situações podem necessitar de cuidados hospitalares, além de interferir no desenvolvimento cognitivo e comportamental por acarretar traumas transitórios ou até mesmo permanentes ${ }^{2}$. Essa questão é um grande desafio às autoridades visto que atinge a população em diversos 
ambientes, em várias fases da vida e em todos os tipos de relações interpessoais ${ }^{2}$.

A violência infantil é praticada das mais diversas formas, sendo que a física compreende qualquer situação que cause dor à mesma; a psicológica envolve situações que geram sofrimento mental; a sexual entende-se como quaisquer atos entre o agressor e o menor, com a finalidade de estimulá-lo sexualmente; e as situações de negligência, que incluem a omissão dos cuidados físicos e/ou mentais necessários a criança $a^{3-5}$.

De acordo com a Organização Mundial da Saúde (OMS), mais de duas mil crianças vêm à óbito todos os dias e a principal causa são os traumas físicos, sendo que as principais vítimas se encontram em uma faixa etária de 0 a 10 anos $^{6}$.

Para ajudar no monitoramento da violência, nos anos 90, o Estatuto da Criança e do Adolescente tornou obrigatória a notificação compulsória de casos suspeitos e/ou confirmados de violência contra menores, com aplicação de multa para quem não a realizar. As notificações são de extrema importância para as políticas públicas e estudos que dimensionam a violência intrafamiliar, visto que é por meio dos dados registrados que é feito um melhor direcionamento de investimentos em programas de vigilância e assistência social a fim de reduzir e/ou evitar o acontecimento da mesma ${ }^{7}$.

Os sinais e sintomas das vítimas podem ser vistos também em ambientes extrafamiliares, como por exemplo, na escola, uma vez que o contato com criança é maior do que em qualquer outro serviço ${ }^{2}$.

Dessa forma, os educadores são de extrema importância nesse contexto devido a convivência diária com os menores, onde devem observar atentamente atitudes que denunciem qualquer tipo de abuso, e assim contribuir na detecção de casos e na prevenção das consequências da violência ${ }^{3,4,8}$. Sendo assim, a escola tem um papel importante na identificação e combate à violência, uma vez que a criança que sofre qualquer tipo de abuso, seja ele físico, psicológico ou sexual, apresenta comportamentos sugestivos, como ansiedade, distúrbio de aprendizado, agressividade, tristeza, comportamento sexual inadequado para a idade, choro sem causa, elevado índice de falta às aulas e distúrbios alimentares. ${ }^{2}$

Baseado neste contexto, 0 exposto estudo teve como objetivo identificar casos suspeitos e/ou confirmados de violência contra crianças em escolas municipais de Ensino Básico em um município de médio porte do Estado de São Paulo.

\section{MATERIAL E MÉTODO}

Trata-se de um estudo descritivo, de caráter transversal e abordagem quantitativa, realizado em 5 escolas municipais de ensino básico (EMEBs), selecionadas por meio de sorteio, no período de agosto de 2018 a julho de 2019. O universo amostral da pesquisa foi composto por 62 professores dessas instituições, e destes, 55 participaram.

Foram incluídos na pesquisa todos os professores que estavam presentes na data da entrevista e aceitaram participar, assinando o termo de consentimento livre e esclarecido. Os critérios de exclusão utilizados foram os professores que estavam de licença médica; afastados de suas atividades laborais; ausentes no dia da entrevista; ou aqueles que não consentiram em participar.

Foi realizado um estudo piloto em uma escola que não entrou para a presente pesquisa, para ajudar no refinamento dos procedimentos de coleta e registro de dados, além de possibilitar o teste dos procedimentos estabelecidos.

A identificação dos casos suspeitos e/ou confirmados de violência contra crianças foi efetuada por meio de um inquérito, respondido pelos professores das instituições. A coleta foi realizada mensalmente por um único pesquisador. Os professores foram estimulados a relatar o que eles observaram de casos suspeitos e/ou confirmados, de forma a analisar a descrição dos casos de violência; a identificação do perfil da criança; a identificação do agressor e o reconhecimento de casos esporádicos e reincidentes.

Os dados colhidos foram quantificados e tabulados no programa Excel para uma análise estatística descritiva, e a fim de identificar quais variáveis poderiam relacionar-se às recidivas dos casos de violência, aplicou-se o teste estatístico do qui-quadrado. A significância estatística foi considerada com o valor de $p<0,05$. As análises foram realizadas no programa BioEstat 5.0.

A presente pesquisa foi aprovada pelo Comitê de Ética e Pesquisa em seres humanos, CAAE 53190015.5.0000.5420.

RESULTADOS

Foram detectados no período da pesquisa, 90 casos suspeitos e/ou confirmados de violência infantil. Dentre as vítimas identificadas pelos professores, $57,8 \%$ eram do sexo masculino, e a idade com maior percentual de vítimas foi a de 5 anos (27,8\%).

$$
\text { Entre os principais suspeitos }
$$

identificados por cometerem as agressões, 
estavam os familiares e pessoas do próprio convívio da vítima. Em $72,2 \%$ dos casos foram os próprios pais, em $8,9 \%$ os avós, em $6,7 \%$ os amigos e em $12,2 \%$ outros agressores.

A forma mais prevalente de violência identificada segundo as características de descrição dos casos foi a negligência $(74,4 \%)$, seguido da agressão física (20\%) e em 5,6\% não havia informações.

Os principais sinais e sintomas percebidos pelos pedagogos foram o piolho $(42,2 \%)$, a cárie dentária $(34,4 \%)$, o uso de roupas inadequadas (12,2\%), a agressividade $(5,6 \%)$, crianças que frequentavam as escolas sem realizar nenhuma refeição $(3,3 \%)$ e a presença de queimaduras de cigarro $(2,2 \%)$. Cerca de $52,5 \%$ dos casos eram reincidentes e em $11,3 \%$ dos casos não foram relatadas tais informações.

A Tabela 1, podemos verificar se as variáveis "sexo" e "tipo de violência" poderiam influenciar na reincidência dos casos de violência. $O$ teste apontou que tais variáveis não foram significativas $(p>0,05)$.

Tabela 1 - Distribuição numérica das variáveis sexo e tipo de violência segundo a recidiva de casos, Araçatuba, 2019.

\begin{tabular}{|c|c|c|c|c|}
\hline Sexo & Reincidente & \begin{tabular}{|l|}
$\begin{array}{l}\text { Não } \\
\text { reincidente }\end{array}$ \\
\end{tabular} & \begin{tabular}{|l|}
$\begin{array}{l}\text { Sem } \\
\text { informações }\end{array}$ \\
\end{tabular} & p-valor \\
\hline Masculino & 26 & 22 & 4 & \multirow{2}{*}{0,4864} \\
\hline Feminino & 21 & 12 & 5 & \\
\hline \multicolumn{5}{|l|}{ Tipo de violência } \\
\hline Negligência & 38 & 23 & 6 & \multirow{3}{*}{0,6444} \\
\hline Agressão Física & 3 & 5 & 2 & \\
\hline Maus tratos & 4 & 3 & 1 & \\
\hline
\end{tabular}

No cenário atual, a violência vem atingindo índices cada vez mais expressivos, atraindo ainda mais a atenção das autoridades brasileiras. Deste modo, nota-se que o desempenho de setores como gestão, política e educação, é de grande relevância para a redução das taxas de agressão ${ }^{3}$.

A presente pesquisa foi executada em ambiente escolar visto que além de ser um local de aprendizado, serve também para a observação e detecção de abusos e/ou comportamentos atípicos e acolhimento para as vítimas ${ }^{9,10}$.

Dos casos notificados neste estudo, entre os gêneros das vítimas não houve discrepância significativa. No entanto, o sexo masculino foi apontado como o mais atingido pela violência infantil, corroborando os estudos de Garbin et al. ${ }^{5}$, Garbin et al. ${ }^{9}$, Veloso et al. ${ }^{10}$, Assis et al. ${ }^{11}$ e Mascarenhas et al. ${ }^{12}$. No entanto, nos estudos de Farias et al. ${ }^{1}$ e Rates et al. ${ }^{13}$, predominaram as vítimas do sexo feminino. Quanto a idade mais prevalente, as vítimas se encontravam em uma faixa etária em torno de 5 anos de idade, assim como em outras análises realizadas ${ }^{1,14}$.
Ao averiguar 0 reconhecimento do agressor, as informações coletadas apontaram os pais como os principais agressores, seguido dos avós, denunciando que os suspeitos de cometer esses atos, na sua grande maioria, são os próprios membros da família ou pessoas que possuem contato próximo com as crianças, o que reforça o risco que os menores correm diariamente. Esses achados também são vistos nos estudos de Farias et al. ${ }^{1}$, Garbin et al. ${ }^{5}$, Rates et al. ${ }^{14}$, Maia et al. ${ }^{15}$, Egryet et al. ${ }^{16}$, Barros et al. ${ }^{17}$, 'Pfeiffer et al. ${ }^{18}$ e Gawryszewski et al. ${ }^{19}$.

Quanto ao tipo de violência, no presente estudo foi demonstrado que a infantil doméstica prevalece sobre outros tipos, sendo que a negligência é a forma mais frequente de se manifestar, seguida pela física. Diversos estudos também indagaram a negligência como o principal modo de violência infantil praticada $^{5,11,13,14,15,18}$. Em controvérsia, outras pesquisas identificaram a agressão física como o tipo mais prevalente ${ }^{1,3,12,19,20}$. A discordância entre os estudos acerca da forma de violência sofrida ocorre devido à dificuldade ou mesmo falta de preparo dos profissionais que possuem contato diário com as vítimas, como os professores, de identificarem os traços característicos de cada uma delas ${ }^{12}$.

A falta de cuidados com a criança é de difícil constatação pelo fato de não expressar traços visíveis, ao contrário da força física, onde o seu uso provoca marcas ${ }^{14}$. Os principais traços de negligência observados pelos professores que levaram a suspeitar da violência foram a presença de piolho, lesões cariosas não tratadas e uso de roupas inadequada. E em menor predominância foi notado sinais de agressividade, falta de alimentação e queimaduras de cigarro, assim como nos achados de Garbin et al. ${ }^{9}$ em que foi notado os mesmos sinais e sintomas, incluindo ainda a presença de ferimentos, equimoses e contusões corporais em áreas difíceis de serem atingidas por quedas.

É importante enfatizar que na presente pesquisa mais da metade dos casos suspeitos e/ou confirmados de abuso eram reincidentes. De acordo com Silva e Mendonça ${ }^{21}$, a alta taxa de violência e sua reincidência está associada à ambientes onde o diálogo é escasso, o nível de estresse nas relações interpessoais é elevado e há uma desvalorização da vida. E ao aplicar o teste estatístico não houve diferença significativa entre as variáveis sexo e tipo de violência segundo a reincidência dos casos.

A violência no âmbito familiar pode acarretar diversas consequências futuras. Egry 
et al. $^{22}$ constaram em seu estudo que há uma transmissão da violência sofrida na infância para a vida adulta. Seguindo este pensamento, tem força de sentido a ideia de Rates et al. ${ }^{14}$ segundo a qual a violência estrutural do âmbito familiar contribuem para a permanência das violações interpessoais nesse ambiente.

Consequentemente, faz-se necessário a elaboração de projetos que priorizem o uso do diálogo de modo a ensinar as crianças, uma vez que ambos tipos de violência deixam memórias no psicológico e acarretam um desenvolvimento pessoal desfavorável. Logo é de extrema importância que exista políticas públicas possam servir de subsídio no combate a violência infantil, uma vez que é complexa a reintegração social desses menores em decorrência do desgaste emocional provocado pelo trauma sofrido ${ }^{10}$.

Por essas razões, os profissionais que possuem contato rotineiro com os menores devem estar sempre atentos e ter uma educação continuada para que possam identificar qualquer indicativo de casos de violência e realizar notificações ${ }^{22,23}$.

CONCLUSÃO

Conclui-se que muitas crianças vêm sofrendo com algum tipo de agressão doméstica e que os sinais e sintomas das vítimas podem ser observados também em ambientes extrafamiliares, como por exemplo, a escola.

REFERÊNCIAS

1. Farias MS, Souza CDS, Carneseca EC, Passos ADC, Vieira EM. Caracterização das notificações de violência em crianças no município de Ribeirão Preto, São Paulo, no período 2006-2008. Epidemiol Serv Saúde, Brasília. 2016;25:799-806.

2. Lloyd M. Domestic Violence and Education: Examining the Impact of Domestic Violence on Young Children, Children, and Young People and the Potential Role of Schools. Front Psychol. 2018;9:2094.

3. Garbin CAS, Lima TJV, Garbin AJI, Rovida TAS, Saliba O. Conhecimento e percepção dos educadores do ensino infantil sobre violência. Rev Ciênc Plural. 2015;1:37-47.

4. Garbin CAS, Queiroz APDG, Costa AA, Garbin NA. Training and knowledge of childhood education teachers on family violence against children. Educar em revista. 2010;2:207-16.

5. Garbin CAS, Araújo PC, Rovida TAS, Rocha AC, Arcieri RM, Garbin AJl. Violence in child population: epidemiological profile of abuses seen in the school environment. Rev Ciênc Plural. 2016;2:41-54.

6. World Health Organization. Global status report on violence prevention (Internet). Geneva: World Health Organization; 2014.
7. Brasil. Ministério da Saúde (MS). Secretaria de Vigilância em Saúde. Departamento de Vigilância de Doenças e Agravos não Transmissíveis e Promoção da Saúde. Sistema de Vigilância de Violências e Acidentes (Viva): 2009, 2010 e 2011. Brasília: MS; 2013.

8. Brino RF, Williams LCA. Capacitação do educador acerca do abuso sexual infantil. Interação psicol. 2003;7(2):1-10.

9. Garbin CAS, Rocha RS, Soares GB, Arcieri RM. Violência infantil e promoção de saúde com pré-escolares. Rev Bras Pesq Saúde. 2016;18:121-28.

10. Veloso LUP, Silva LCL, Sousa CR, Rodrigues PL. Perfil de violência em crianças de 0 a 9 anos atendidas em um hospital público. Rev Enferm UFPI. 2015;4:97-105.

11. Assis SG, Avanci JQ, Pesce RP, Pires TDO, Gomes DL. Notificações de violência doméstica, sexual e outras violências contra crianças no Brasil. Cienc saude coletiva. 2012; 17:2305-17.

12. Mascarenhas MDM, Malta DC, Silva MMAD, Lima CM, Carvalho MGOD, Oliveira VLAD. Violência contra a criança: revelando o perfil dos atendimentos em serviços de emergência, Brasil, 2006 e 2007. Cad Saúde Publica. 2010; 26:347-57.

13. Rates SMM, Melo EMD, Mascarenhas MDM, Malta DC. Violência infantil: uma análise das notificações compulsórias, Brasil 2011. Ciênc saúde coletiva. 2015;20:655-65.

14. Apostólico MR, Nóbrega CR, Guedes RN, Fonseca RMGS, Egry EY. Características da violência contra a criança em uma capital brasileira. Rev Latina-Am Enfermagem. 2012; 20:266-273.

15. Maia JN, Ferrari RAP, Gabani FL, Tacla MTGM, Reis TB, Fernandes MLC. Violência contra criança: cotidiano de profissionais na atenção primária à saúde. Rev Rene. 2016;17:593-601.

16. Egry EY, Apostolico MR, Morais TCP, Lisboa CCR. Coping with child violencein primary care: how do professionals perceive it? Rev Bras Enferm. 2017;70:113-19.

17. Barros ASD, Freitas MFQ. Violência doméstica contra crianças e adolescentes: consequências e estratégias de prevenção com pais agressores. Pensando Fam. 2015;19:102-114.

18. Pfeiffer L, Rosário NA, Nunes LC. Violência contra crianças e adolescentes - proposta de classificação dos níveis de gravidade. Rev Paul Ped. 2011;29:477-482.

19. Gawryszewski VP, Valencich DMDO, Carnevalle CV, Marcopito LF. Maus-tratos contra a criança e o adolescente no Estado de São Paulo, 2009.Rev assoc méd bras. 2012;58:659-65. 
20. Carvalho ACR, Barros SGD, Alves AC, Gurgel CA. Maus-tratos: estudo através da perspectiva da delegacia de proteção à criança e ao adolescente em Salvador, Bahia. Cienc saude coletiva. 2009;14:539-46.

21. Silva LS, Mendonça KML. A violência escolar em matérias de jornal: um imaginário construído em Belém-PA. Comun Educ. 2015;20:39-49.

22. Egry E, Silva M, So K, Apostólico M. Limites e potencialidades dos profissionais, instituições e políticas públicas para o enfrentamento da violência doméstica infantil na Atenção Primária de Saúde: visão dos gestores. CIAIQ. 2016;02.

23. Abreu PTR, Souza Costa IF, Galvão A, Paula Souza AC, Zocratto KBF, Oliveira CAS. Abuso físico infantil: vivências e atitudes de estudantes de Odontologia. Rev ABENO. 2017; 17:107-19.

\section{CONFLITO DE INTERESSES}

Os autores declaram não haver conflitos de interesse

\section{AUTOR PARA CORRESPONDÊNCIA}

\section{Cléa Adas Saliba Garbin}

NEPESCO - Núcleo de Pesquisa em Saúde Coletiva Faculdade de Odontologia de Araçatuba UNESP - Univ Estadual Paulista

Departamento de Odontologia Preventiva e Restauradora R. José Bonifácio, $\mathrm{n}^{\circ} 1193$ - Vila Mendonça 16015-050 - Araçatuba-SP - Brasil

Tel. (55 018) 3636-2824

E-mail: clea.saliba-garbin@unesp.br 